\title{
Cueless blindsight
}

\section{Petra Stoerig*}

Institute of Experimental Psychology, Heinrich-Heine-University Dusseldorf, Dusseldorf, Germany

\section{Edited by:}

Stephen A. Engel, University of

Minnesota, USA

Reviewed by:

Antony B. Morland,

University of York, UK

Holly Bridge, University of Oxford, UK

*Correspondence:

Petra Stoerig, Institute of Experimental Psychology II, Heinrich-Heine-

University, Bldg. 23.03,

Universitaetsstrasse 1, 40225

Dusseldorf, Germany.

e-mail: petra.stoerig@uni-duesseldorf.de
The term blindsight describes the non-reflexive visual functions that remain or recover in fields of absolute cortical blindness. As visual stimuli confined to such fields are subjectively invisible, they are customarily announced by visible or audible cues that inform the patients when to respond. The pervasive use of cueing has spawned the widely held assumption that sight and blindsight differ in that only blindsight requires cueing. To test this assumption, we measured detection of auditorily cued and un-cued stimuli in three hemianopic patients. Stimuli fell onto the photosensitive retina of the subjectively blind field, onto the objectively blind optic disc, and, in one patient, into a region where they evoked impoverished conscious sight. Regardless of whether cues were given, performance was highly significant in the latter region of poor sight, clearly above chance in the subjectively blind field, and random in the optic disc control condition. Moreover, cues enhanced detection only in the relatively blind field. Showing that blindsight performance persists when cues are omitted, the results imply that non-reflexive responses can be initiated in the absence of both stimulus awareness and perceptible cues.

Keywords: blindsight, cueing, stimulus awareness, function of conscious sight, hemianopia, cortical blindness

\section{INTRODUCTION}

Patients who suffered lesions of the primary visual cortex can detect, localize, and discriminate targets when 'forced' to 'guess' whether, where or which stimulus has briefly been presented to their cortically blind field (Weiskrantz, 1986; Stoerig and Cowey, 1997 for reviews). This counterintuitive ability to respond to subjectively invisible stimuli has become widely known as blindsight (Weiskrantz et al., 1974). By virtue of informing the debate of the function(s) (Dennett, 1991; Block, 1995) as well as the neural bases of conscious sight (Zeki, 2001; Tong, 2003), blindsight has become one of the most widely discussed examples of implicit functions in neurological patients. Subjectively invisible stimuli have been reported to elicit reflexive responses including pupillary (Weiskrantz et al., 1999) and electrodermal (Zihl et al., 1980) responses. Moreover, subjectively invisible stimuli have been shown to modulate voluntarily initiated responses to visible targets presented to the sighted hemifield of hemianopic patients (Marzi et al., 1986; Corbetta et al., 1990); related findings come from normal sighted participants where experimental manipulations such as backward masking and Transcranial Magnetic Stimulation (Breitmeyer et al., 2004) are used to prevent stimulus awareness. In marked contrast, the initiation of non-reflexive responses is commonly held to require stimulus awareness. Consequently, ever since Pöppel et al. (1973) introduced forced-choice methods to study the non-reflexive residual visual functions that remain in the cortically blind fields of human patients, researchers have given visible or audible cues to inform the patients when to respond. This customary cueing led to the conclusion that "Blindsight subjects have to be prompted or cued to give their better-than-chance "guesses". [...] Without such cues, the subject simply fails to respond" (Dennett, 1991, p. 328).

If blindsight thus differed from sight by virtue of requiring consciously accessible cues, the subjectively invisible blind-field stimuli would inform the responses and thereby permit the often highly significant performance; however, they would not suffice to elicit them. To learn whether blindsight fails without cues, we here tested detection of visual stimuli in three hemianopic patients who had suffered unilateral destruction of the primary visual cortex as adults.

\section{MATERIALS AND METHODS PATIENTS}

About six years before the present experiments began the three male participants suffered unilateral vascular lesions in the territory of the posterior cerebral artery. The damage affected predominantly the primary visual cortex in HK and WF whose lesions are of ischemic origin; BT's much larger defect was caused by removal of an arteriovenous malformation (Figure 1A). All three patients had extensive prior experience with tests of their residual visual functions (Stoerig et al., 2002; Stoerig, 2006). They gave informed consent to the experiments that were approved by the University Ethics Committee.

\section{VISUAL FIELD PERIMETRY}

WF has an incomplete hemianopia, while HK and BT both have a complete hemianopia with macular sparing extending up to $5^{\circ}$ eccentricity in $\mathrm{HK}$ and $2^{\circ}$ in BT. Plots for the left eye that was used for further testing are shown in Figure 1B. They are based on a combination of static and dynamic perimetry performed at a Tübingen perimeter (Oculus, Wetzlar, Germany). The $116^{\prime}, 320 \mathrm{~cd} / \mathrm{m}^{2}$ white stimulus was moved slowly from the periphery towards the central fixation dot $\left(30^{\prime}\right.$, red), or from the blind towards the seeing part of the field; the white background had a luminance of $10 \mathrm{~cd} / \mathrm{m}^{2}$. Following delineation of visual field borders, the same stimulus was presented for $200 \mathrm{~ms}$ at closely spaced positions within the field defect, to detect any islands of vision. Within the shaded regions shown in Figure 1, this stimulus did not yield detection responses. Nevertheless, HK who has recovered some poor sight in the central 
A

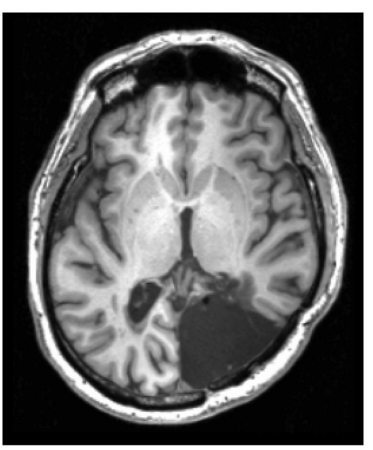

B

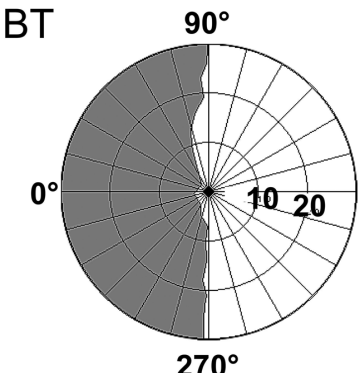

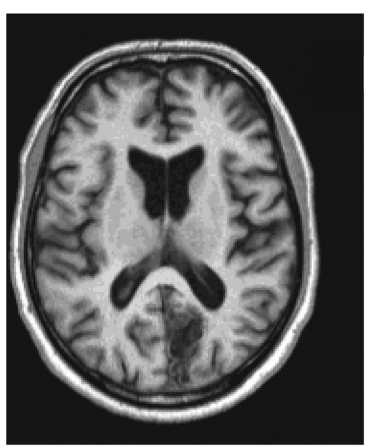

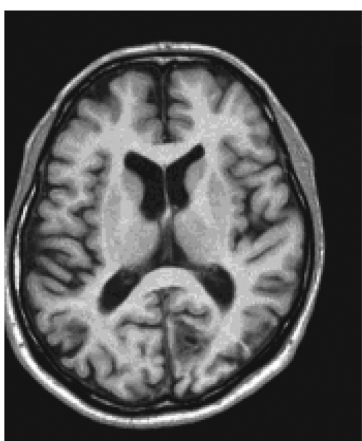

HK

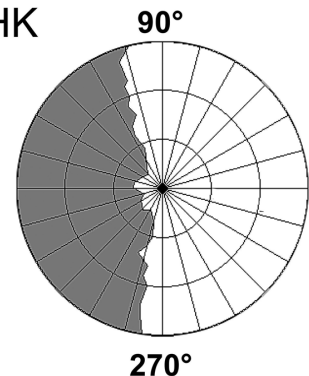

$270^{\circ}$
WF $90^{\circ}$

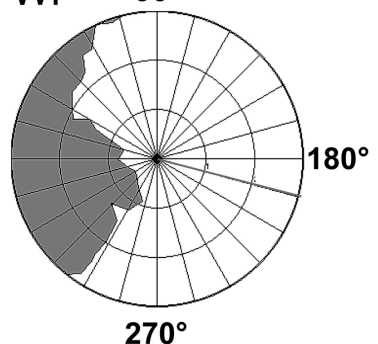

C

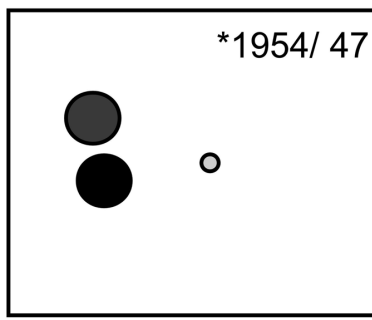

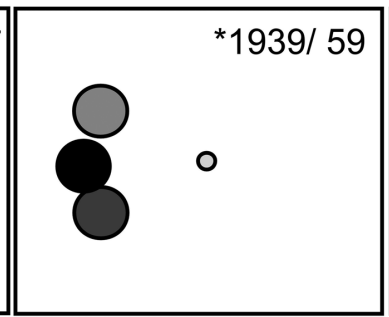

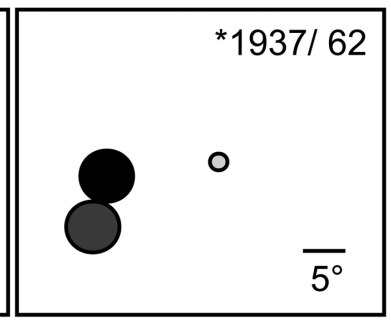

D

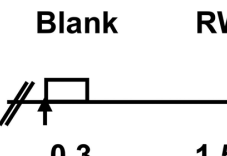

1.5

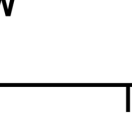

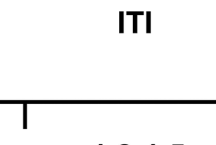

1.2-1.5

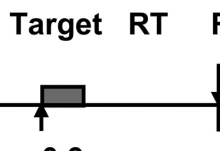

0.3

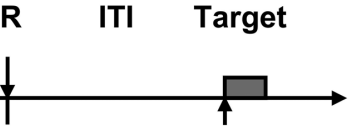

1.2-1.5 $0.3 \mathrm{~T}[\mathrm{~s}]$

FIGURE 1 | Patient and experimental information. (A) T1-weighted magnetic resonance images depicting the lesions in the right occipital lobe. (B) The visual fields of the left eye are plotted to an eccentricity of $\pm 30^{\circ}$. They were mapped at a Tübinger perimeter (Oculus, Wetzlar, Germany) using a combination of dynamic and static (200 ms presentation) perimetry. Stimuli were white $116^{\prime}, 320 \mathrm{~cd} / \mathrm{m}^{2}$ circular targets presented on a $10 \mathrm{~cd} / \mathrm{m}^{2}$ neutrally white background. (C) The positions used for testing detection are shown on a cartoon screen. They had the same negative contrast $(-\log 0.61)$ at all positions; the different gray levels symbolize the density of blindness, with black corresponding to the optic disc, dark gray to the absolute, light gray to the relative defect positions, respectively. The participants' year of birth and age at lesion are indicated. (D) Time course of event types. Only in the cued condition were both target and blank stimulus presentations announced by a brief beep at stimulus onset. RW, response window; ITI, inter-trial interval; $R$, response; $R T$, response time. region of his upper left hemifield, often reports vague shadows in response to targets of high contrast. More peripheral regions as well as the lower quadrant remained experientially blind.

\section{EXPERIMENTAL PROCEDURE}

During testing, with the head supported by a chin rest, subjects fixated a central dot on a computer monitor (Philips, refresh rate $50 \mathrm{~Hz}$ ) that subtended $34.3 \times 26.8^{\circ}$ at the viewing distance of $67 \mathrm{~cm}$. It was encased in a black felt-lined box to minimize cues from light scatter; the eye with the defect in the nasal hemifield was covered with a patch to restrict presentation to the smaller defect in the temporal hemifield (Teuber et al., 1960) and to avoid artifacts from compensatory squints. The target was a $16 \mathrm{~cd} / \mathrm{m}^{2}$ gray disk $5.3^{\circ}$ in diameter and appeared for $300 \mathrm{~ms}$ on the neutral white background of the screen $\left(65 \mathrm{~cd} / \mathrm{m}^{2}\right)$. Target positions, between 12.6 and $18.7^{\circ}$ off fixation, were adjusted to the individual defect. In WF and BT, one position in the blind field was tested, but two, one in the upper, one in the lower quadrant, were used in $\mathrm{HK}$, so as to test the effect of cueing on poorly visible (HK's region of poor sight, $\left.\mathrm{HK}_{\mathrm{u}}\right)$ as well as subjectively invisible stimuli $\left(\mathrm{HK}_{1}\right)$. To control for artifacts from target reflections, in all patients stimuli were additionally presented on the natural blind spot within the 
blind field; its position was estimated based on the position of the blind spot in the other eye and confirmed with Scanning Laser Ophthalmoscopy (Rodenstock, Ottobrunn, Germany). Stimulus positions, indicated in Figure 1C, were always tested in separate blocks of trials. To avoid a possible impact on criterion, the participants were not informed about the blind spot control tests which were performed in random alternation with the blind field tests. However, because they inquired where targets would appear, participants were told whether the target would be presented to the upper or lower blind field.

As chance level performance in un-cued conditions would be uninformative if the patients responded no better than expected by chance guessing in cued conditions, and to compare performance in cued and un-cued tests, data were collected without cues as well as with both target and no target ('blank') stimuli announced by $500 \mathrm{~Hz}, 100 \mathrm{~ms}$ beeps at stimulus onset. Cued and un-cued trials were never mixed in the same block. In both conditions, the patients' task was to maintain fixation faithfully, and to press a button on a response box (RB-620, Cedrus, San Pedro, CA, USA) to signal 'target'. Blank stimuli required no response. Target probability was $40 \%$. In neither condition were the participants informed about target probability, and were only instructed to respond 'at least a few times to allow for statistical evaluation of the data'. As all participants had performed significantly better than expected by chance guessing in previous experiments (see above), testing began with the un-cued condition. Six to eight blocks of 120 uncued stimuli, 48 targets, were presented per patient and position before cued tests were introduced. Testing then continued with cued and un-cued blocks interleaved. Block length was halved to 60 stimuli, of which 24 were targets, during this second period, to alleviate the strain of prolonged fixation. Testing continued in pseudo-random alternation until a minimum of nine blocks was collected per patient, position, and condition.

Superlab Pro (ProGamma, Groningen, The Netherlands) was used as programming platform. Each target presentation was succeeded by a 1,500 ms response window which was terminated by a response. Blank trials were also followed by $1,500 \mathrm{~ms}$ response windows. Intertrial intervals (ITIs) ranging from 1,200 to $1,500 \mathrm{~ms}$ filled the remainder of each block (Figure 1D). We monitored fixation throughout with an infrared remote camera system (IView, SensoriMotorInstruments, Teltow, Germany), and recorded eyemovements in relation to the stimuli whose presentation provided the triggers.

\section{DATA ANALYSIS}

Participants signaled target stimulus presentations only, and only responses that occurred within target response windows were counted as correct. In our first analysis that compared cued and uncued performance, these hits were compared to the false responses given within the blank stimulus response windows (false alarms). The chi-square test was used to assess whether performance differed from random. This analysis corresponds to procedures used in cued tests where responses are confined to the response windows. To derive a single non-parametric index of detection to allow comparisons across conditions, the False Alarm Rate (FAR) was deducted from the Hit Rate (HR). Like HR and FAR, the resultant measure HR-FAR accounts for response bias.
As this standard analysis ignores the responses given during target and blank stimulus presentations and ITIs in the un-cued condition, all responses were taken into account in a second analysis. It was performed only on the un-cued data, because cues prevent patients from responding outside of the response windows. For this second analysis, the observed percentage correct, that is the percentage of target presentations that yielded a response within the $1,500 \mathrm{~ms}$ target response windows, was compared to the percentage correct value expected on the basis of randomly occurring responses. This 'chance' value was calculated as the fraction of all responses that corresponded to the sum of all target response windows divided by the total length of the series. In other words, if all target response windows together made up 25\% of the total series' duration, approximately $25 \%$ of all responses given should be correct on the basis of random guessing; chance performance would thus be approximately $25 \%$ correct. As this procedure estimates the expected ('chance') values on the basis of the collected data, it also accounts for response bias. The Wilcoxon Signed Rank test for dependent samples (Wilcoxon, 1945) was used to compare observed and expected values over the blocks collected for each stimulus position and patient. Alpha was adjusted for multiple comparisons.

\section{RESULTS}

\section{THE CUED CONDITION}

When audible cues announced the target and blank stimuli, performance was statistically significant in all patients provided the stimuli were presented to the photosensitive retina of the blind field rather than the blind spot. Figure 2A depicts the Hit and False Alarm Rates separately for each patient and position. The chi-square values and their corresponding $P$-values indicate that performance was best in HK's field of poor sight $\left(\mathrm{HK}_{\mathrm{u}}\right)$, but still significant at $P=0.01$ or lower in the absolute defects. However, targets presented so as to fall onto the optic disc yielded chance level performance.

\section{PERFORMANCE IN THE UN-CUED CONDITION}

When the analysis was restricted to responses occurring in the response windows, detection performance again significantly exceeded chance level as long as stimuli were presented to the photosensitive retina of the blind field (Figure 2B). As in the cued condition, stimulation of HK's upper quadrant yielded the best results, while the blind spot control condition produced chance level results.

Cued and un-cued conditions are directly compared in Figure 3. Figure 3A depicts the mean differences between Hit and False Alarm Rates (HR-FAR) for all patients and positions. This index was compared between conditions to assess the effect of cueing. A statistically significant effect of cueing was found for HK's performance in the upper quadrant. Remarkably, no statistically meaningful effect was found for any other position. Cueing thus improved detection only where targets generated weak stimulus sensations.

Mean response rates for cued and un-cued conditions are shown in Figure 3B. Probably due to the patient's ignorance of target probability, response frequency per block varied widely, and ranged from 1 to 354 responses in the un-cued condition. Overall, however, response frequencies were lower in the latter than in the cued 


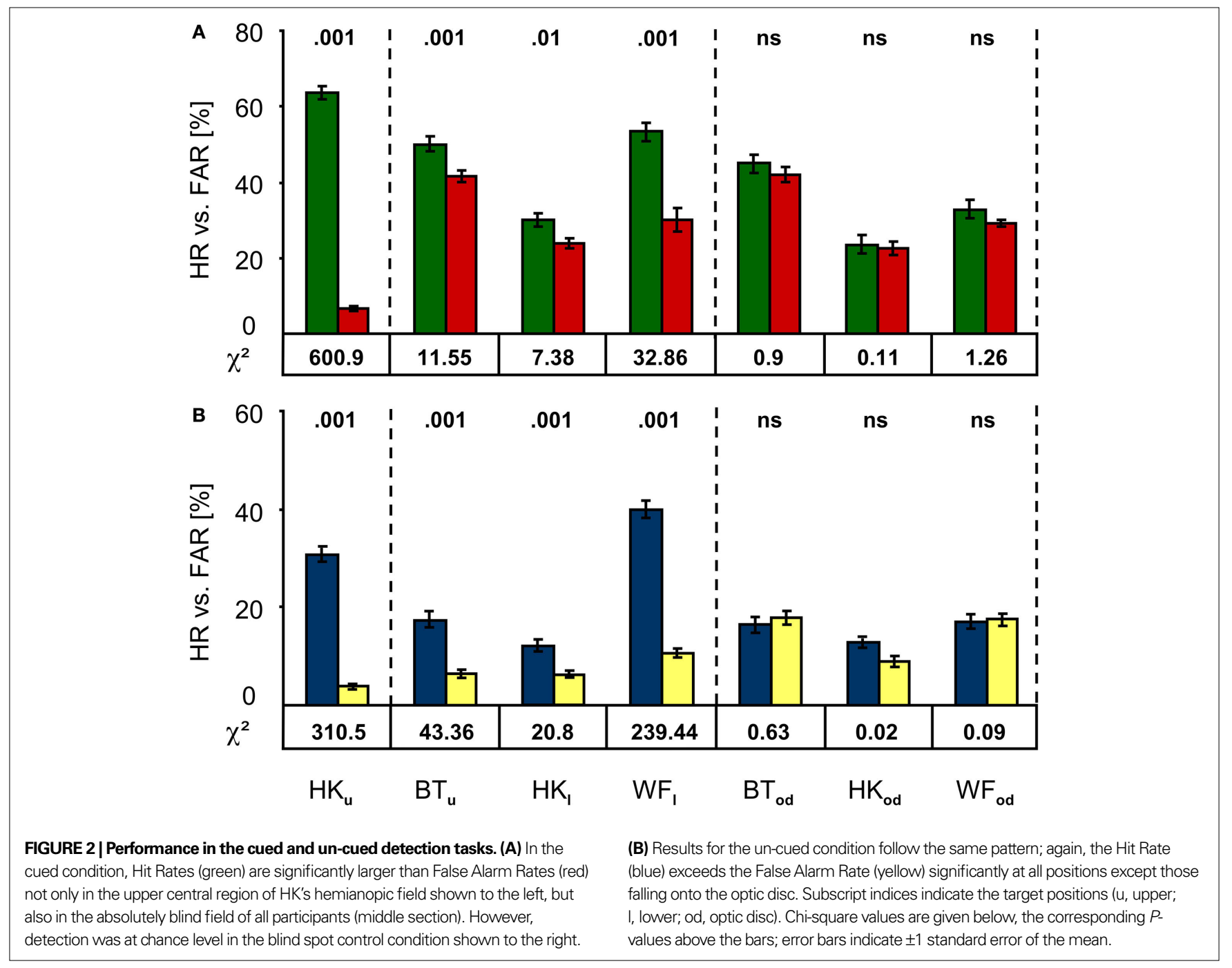

condition. This difference was significant in all but one case $\left(\mathrm{HK}_{\mathrm{od}}\right)$. Regardless of whether or not cues announced stimulus presentations, no statistically meaningful difference was found when response rates recorded from the blind field were compared to those from the optic disc positions. A significant difference for the uncued condition only was seen when HK's response frequency was compared for the upper and lower hemifield $(Z=-2.76 ; P=0.004)$. Whereas cues thus failed to enhance target detectability at all positions except in HK's region of poor sight, they did markedly increase the number of responses; omission of cues clearly diminished the participants' propensity to respond.

As responses may occur at any time during the block when cues are omitted, for each block we also calculated response frequencies for ITIs and (target and blank) stimulus presentation time windows in addition to target and blank stimulus response windows. The recorded response frequency distributions were compared to those expected to fall into these same event type time windows on the basis of response frequency and block duration. For each time window, observed frequencies were compared to the expected 'chance' values derived from the overall response frequency for the same block. As shown in Figure 4, the results are consistent across patients. When the photosensitive retina of the subjectively blind field was stimulated, only the responses given in target stimulus response windows exceeded the expected values. The reverse held true for all other time windows whenever a contrast reached the statistical criterion. No significant difference was found for the optic disc control.

At HK's upper field position, the correct responses exceeded the expected values by $\sim 50 \%$. In contrast, observed response frequency was significantly lower than expected on the basis of proportionate event duration in all other time widows (Figure 5A).

Figure 5B depicts an example of HK's fixation during a typical block of 120 trials in relation to the target's position in the upper hemifield. In this block, HK scored $46 \%$ correct; the corresponding expected value was $18 \%$. Although saccades were predominantly directed to the upper left, their amplitude reached a maximum of $1.9^{\circ}$ to the left and $2.8^{\circ}$ upward. This would not suffice to bring the stimulus partially in the normally sighted field.

\section{DISCUSSION}

All three participants performed better than expected on the basis of random guessing as long as their photosensitive retina was stimulated. This held true in the cued as well as the un-cued condition. 


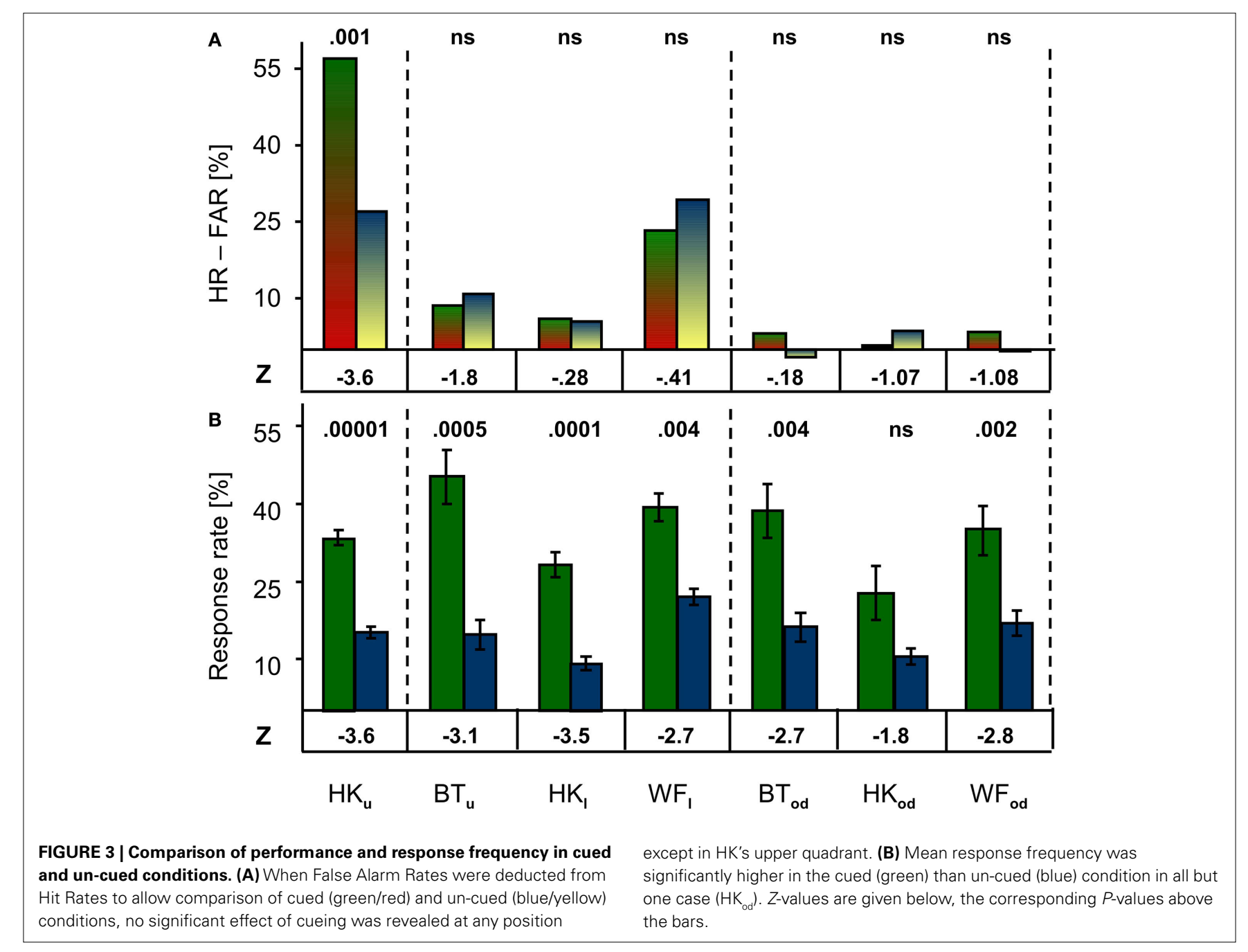

In both, performance was best in HK's region of poor sight, but at chance level when targets fell onto the optic disc. Strikingly, blindsight not only persisted when cues were omitted, but was no worse than in the cued condition. Although cueing heightened response probability, it enhanced performance only in HK's region of poor sight.

\section{CAN ARTIFACTS EXPLAIN THE RESULTS?}

In view of the continuing debate of a role of artifacts in blindsight research and the unexpectedness of the results (we did not expect cueless blindsight to be possible), we need to ask whether the data can be explained on the basis of the usual artifactual suspects. These include (1) intra- or extraocular light scattered into the sighted hemifield, (2) eye movements towards the target positions, and (3) particularly conservative responses criteria adopted when the blind field is tested (e.g. Campion et al., 1983). (1) Stray-light is generally less prominent for stimuli of negative contrast. Here, the targets had a contrast of $-0.61 \log$ to the background, which is considerably lower than the value of 2-3 log previously found necessary for $2^{\circ}$ stimuli of positive contrast presented on the blind spot to be rendered detectable on the basis of stray-light (Stoerig and Cowey, 1989). Moreover, presentation of the target stimuli in the region of the optic disc yielded no evidence of detection. Stimuli $5.3^{\circ}$ in diameter will most likely not be confined to the optic disc throughout the blocks when, as here, no retinal stabilization is used. That the patients' nevertheless failed to detect the stimuli at this position argues against a possible artifact from light scatter, and indicates that whatever part of the stimuli fell outside of this receptor-free region was insufficient to generate better-than-chance performance. (2) The stability of fixation over blocks that could last more than 6 min reflects the participants' prior experience with blindsight tests as well as their high motivation to perform correctly. Eye-movements toward the target positions were confined to regions too small to bring the stimulus across the border of the sighted field. Again, the fact that performance failed to meet the statistical criterion when the stimuli were presented onto the optic disc speaks against a role of eye-movements. Moreover, there was no systematic difference between the eccentricity of blind field and optic disc positions, and detection should have favored the positions closer to the normal sighted field if it was enabled by eye-movements. (3) Any differences in the participants' decision criterion would have to be modulated by target positions within the affected hemifield rather than between the sighted and blind hemifield; this appears unlikely especially with regard to the blind spot vs. abso- 


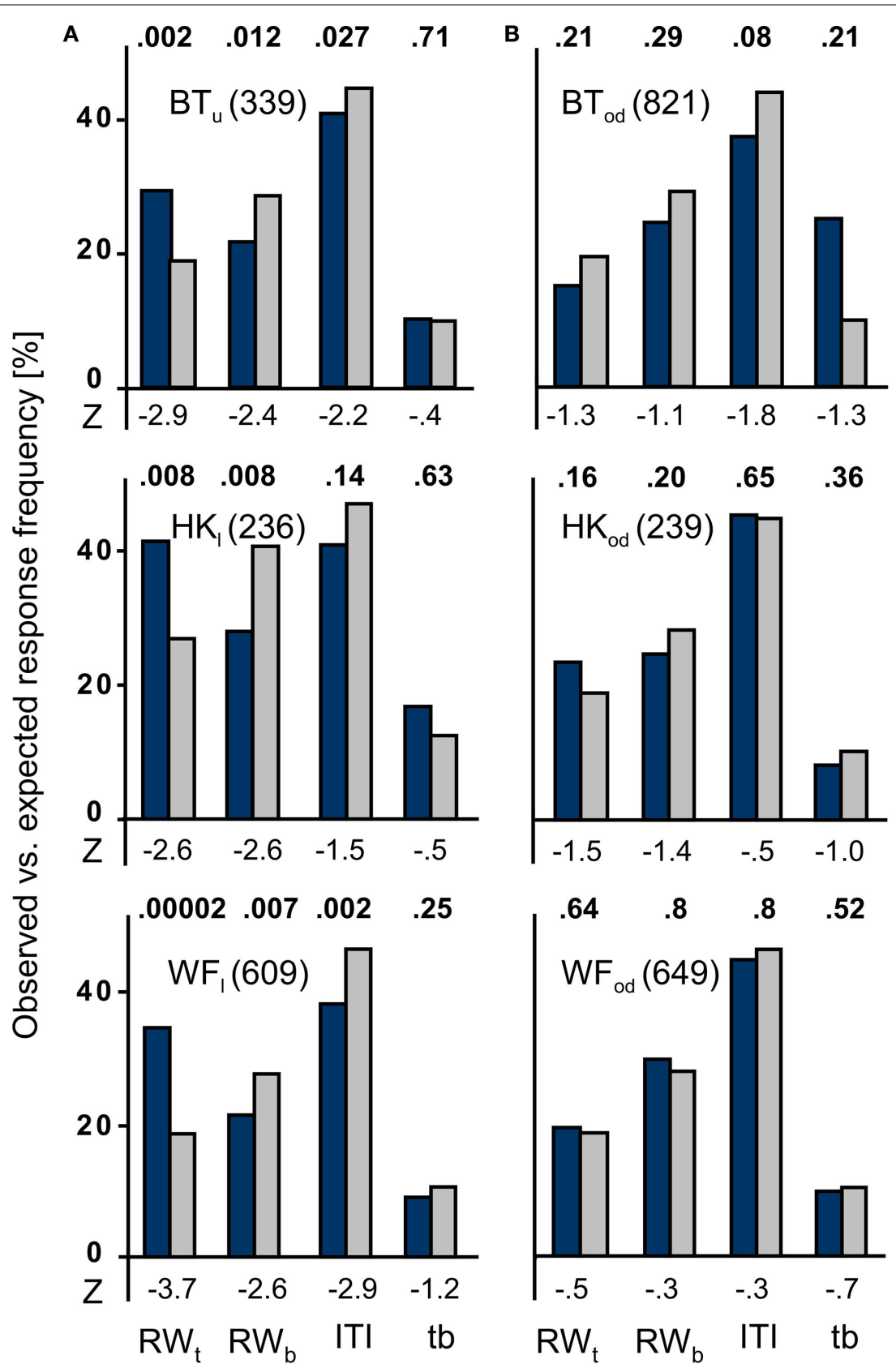

FIGURE 4 | Observed vs. expected response frequencies in the un-cued condition. The blue bars represent the mean observed responses, the gray bars the expected 'chance' values that mirror the proportionate duration of event type time windows. The numbers given behind the participants' initials indicate the total number of responses recorded for the patient at the position indicated by the subscript index. (A) Data for the subjectively blind field are given in the left column. Note that only the correct responses, i.e. those that occurred in target stimulus response windows, exceed the expected values. In the other three intervals, any statistically significant difference favours the expected values. (B) In the blind spot control tests shown to the right, no statistically meaningful difference between observed and expected values was observed. RWt, target response window; RWb, blank stimulus response windows; ITI, intertrial interval; tb, target and blank stimulus presentation time windows. lutely blind field positions. In sum, the experimental conditions, optic disc tests, and fixation stability (note that the example shown in Figure 5B is indeed typical), render an explanation based on the discussed artifacts unlikely. Moreover, attempting to explain the pattern of results in their terms would lead one to expect better performance in the cued condition. Cueing provides temporal scaffolding for eye-movements and alerts participants to possible target presentations; however, it made no difference to performance in the absolute field defects. In our blindsight experienced participants, cueless blindsight was no worse than its cued counterpart. 


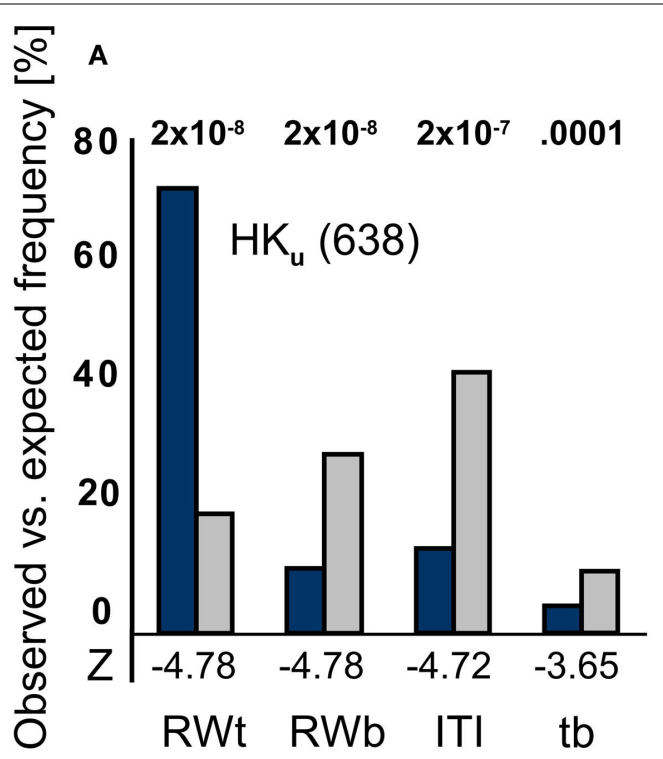

B

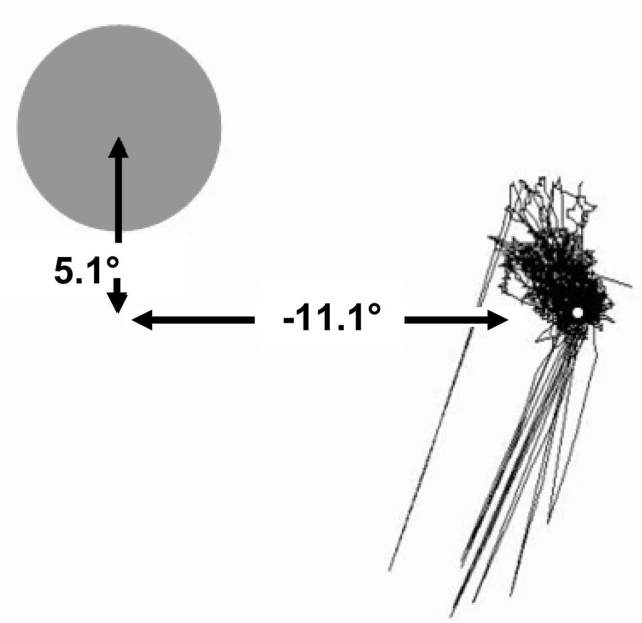

FIGURE 5 | Mean observed vs. expected response frequency and fixation for $\mathbf{H K}_{\mathrm{u}}$. (A) $\mathrm{HK}_{\mathrm{u}}$ 's responses occurred significantly more often than expected by chance in the target response windows, and significantly less often in all other event type time windows. The first $P$-value is the same for target and blank stimulus response windows. Notation as in Figure 4. (B) Eye movements recorded over a typical block of 120 trials in the un-cued condition are shown in relation to the stimulus position in the upper quadrant. Lines toward the lower left reflect blinks.

\section{PREVIOUS STUDIES OF UN-CUED PERFORMANCE IN HEMIANOPIC FIELDS}

Kentridge et al. (1999) used a spatial 2-alternative forced choice (2AFC) task with a hemianope who suffered destruction of the left primary visual cortex at age 8. GY, subject of many studies, indicated the target position as well as whether he had any awareness of an event occurring during the trial. A visual cue at fixation could signal their $400 \mathrm{~ms}$ target, preceding or succeeding it by 1,500 or $500 \mathrm{~ms}$. On 50 trials per 100 trial block no cue was given. Each trial lasted for $10 \mathrm{~s}$, and cues, when they occurred, could do so at five equiprobable and evenly spaced positions during this period.
With or without cues GY performed practically at ceiling when blind-field stimulus contrast was $98 \%$, but profited especially from cues preceding the target at 43 and $22 \%$ contrast; detection of nearthreshold 3\% contrast targets presented to the sighted field was also clearly enhanced by cues. GY reports awareness of sufficiently salient stimuli presented to the upper quadrant of his cortically blind field (for examples, see Barbur et al., 1980, 1993; Weiskrantz et al., 1995; Zeki and Ffytche, 1998; Stoerig and Barth, 2001). In Kentridge et al.'s study where stimuli were presented to this region, GY's awareness reports, like performance, depended on stimulus contrast; they were 98, 26 and $4 \%$ for the high, medium, and low contrast conditions respectively. Because of his stimulus awareness, GY's data are best compared to those of $\mathrm{HK}_{\mathrm{u}}$ who reported vague sensations when tested with $\sim-75 \%$ contrast targets and, like GY at medium and low contrast, profited from cues. Together, GY's and HKu's results suggest that responses to stimuli that generate weak and unreliable sensations profit from cues, or, in the words of Kentridge et al., that 'residual vision is subject to modulation by attentional processes, or arousal, associated with temporal cueing' (p. 479). Detection of stimuli that yield easier-to-detect sensations - GY at 98\% contrast - however, may not profit because it does not require cues. Our present data go beyond those of Kentridge et al.'s. We used a different task (detection rather than 2AFC detection plus localization) and a vastly longer interval of temporal uncertainty (up to $>6$ min rather than $10 \mathrm{~s}$ max.), and we tested three participants in regions of absolute cortical blindness as well as on the optic disc. Our results demonstrate not only that cueing does not affect performance in the control condition but, most noteworthy, that detection of subjectively invisible targets does not profit from cueing. In this regard blindsight resembles normal sight, but neither normal near-threshold vision (for other differences see Azzopardi and Cowey, 1997), nor HK's poor sight.

Two further studies, both on hemianopic monkeys, compared cued and un-cued forced-choice detection and saccadic localization of high-contrast targets. Moore et al. (1995) reported that performance in the blind field of adult-lesion animals deteriorated once target presentation was no longer signaled by a visible cue. In contrast, infant lesion monkeys performed the same task at high levels of precision regardless of whether cues were given (Moore et al., 1996). The authors suggested that their adult-lesion monkeys' behavior resembled human blindsight evoked by subjectively invisible targets, but interpreted their infant-lesion monkeys' success as evidence for residual vision that they owed to their younger system's greater plasticity (Payne et al., 1996). Viewed in the light of the present finding of cueless blindsight in adult-lesion humans and their implications as outlined above, one might conclude that the infant-lesion animals indeed had reasonably good sight in the affected hemifield, because, like GY, they performed very well without cues at high contrast. Alternatively, they could have had better blindsight than the adult-lesion monkeys because their younger systems' greater plasticity allowed for more efficient perceptual learning even in the absence of residual conscious sight. By entering the increasing evidence of perceptual learning in cortically blind fields (Zihl, 1980; Bridgeman and Staggs, 1982; Sahraie et al., 2006; Stoerig, 2006, 2008; Huxlin, 2008; Huxlin et al., 2009) into the equation, we could also tentatively resolve the contrary 
findings in adult-lesion primates. If our participants have, over the course of several years of practice, learned to exploit their blindsight to an extent that allows them to perform equally well with and without cues, the adult-lesion monkeys may not have had sufficient blindsight experience to reach that level. The improved un-cued performance one adult-lesion animal showed in the re-test (Moore et al., 1996) would agree with this interpretation.

\section{FUNCTION OF STIMULUS AWARENESS}

Whereas poor sight profited from cues, cues did not enhance, let alone enable blindsight in our participants. Stimuli presented to regions of absolute cortical blindness can thus prompt, rather than merely modulate, non-reflexive responses. While it is true that our participants responded significantly less often in the un-cued condition, and that their responses were 'forced' button presses that lack the flexibility attributed to planned movements

\section{REFERENCES}

Azzopardi, P., and Cowey, A. (1997). Is blindsight like normal, near-threshold vision? Proc. Natl. Acad. Sci. U.S.A. 94, 14190-14194.

Barbur,J.L., Ruddock, K.H., andWaterfield, V. A. (1980). Human visual responses in the absence of the geniculo-calcarine projection. Brain 103, 905-928.

Barbur, J. L., Watson, J. D., Frackowiak, R. S., and Zeki, S. (1993). Conscious visual perception without V1. Brain 116, 1293-1302.

Block, N. (1995). On a confusion about a function of consciousness. Behav. Brain Sci. 18, 227-287.

Breitmeyer, B. G., Ro, T., and Ogmen, H. (2004). A comparison of masking by visual and transcranial magnetic stimulation: implications for the study of conscious and unconscious visual processing. Conscious. Cogn. $13,829-843$.

Bridgeman, B., and Staggs, D. (1982). Plasticity in human blindsight. Vision Res. 22, 1199-1203.

Campion, J., Latto, R., and Smith, Y. M. (1983). Is blindsight an effect of scattered light, spared cortex, and nearthreshold vision? Behav. Brain Sci. 6, 423-448.

Churchland, P. M. (1984). Matter and Consciousness. Cambridge, MA, MIT Press.

Corbetta, M., Marzi, C. A., Tassinari, G., and Aglioti, S. (1990). Effectiveness of different task paradigms in revealing blindsight. Brain 113, 603-616.

Dennett, D. C. (1991). Consciousness Explained. Boston, Little, Brown and Company.

Huxlin, K. R. (2008). Perceptual plasticity in damaged adult visual systems. Vision Res. 48, 2154-2166.

Huxlin, K. R., Martin, T., Kelly, K., Riley, M., Friedman, D. I., Burgin, W.S., and
Hayhoe, M. (2009). Perceptual relearning of complex visual motion after $\mathrm{V} 1$ damage in humans. J. Neurosci. 29, 3981-3991.

Kentridge, R. W., Heywood, C. A., and Weiskrantz, L. (1999). Effects of temporal cueing on residual visual discrimination in blindsight. Neuropsychologia 37, 479-483.

Marzi, C. A., Tassinari, G., Aglioti, S., and Lutzemberger, L. (1986). Spatial summation across the vertical meridian in hemianopics: a test of blindsight. Neuropsychologia 24, 749-758.

Moore, T., Rodman, H. R., Repp, A. B., and Gross, C. G. (1995). Localization of visual stimuli after striate cortex damage in monkeys: parallels with human blindsight. Proc. Natl. Acad. Sci. U.S.A. 92, 8215-8218.

Moore, T., Rodman, H. R., Repp, A. B., Gross, C. G., and Mezrich, R. S. (1996). Greater residual vision in monkeys after striate cortex damage in infancy. J. Neurophysiol. 76, 3928-3933.

Payne, B. R., Lomber, S. G., Macneil, M. A., and Cornwell, P. (1996). Evidence for greater sight in blindsight following damage of primary visual cortex early in life. Neuropsychologia 34, 741-774.

Pöppel, E., Held, R., and Frost, D. (1973). Residual visual function after brain wounds involving the central visual pathways in man. Nature 243, 295-296.

Sahraie, A., Trevethan, C. T., MacLeod, M. J., Murray, A. D., Olson, J. A., and Weiskrantz, L. (2006). Increased sensitivity after repeated stimulation of residual spatial channels in blindsight. Proc. Natl. Acad. Sci. U.S.A. 103, 14971-14976.

Stoerig, P. (2006). Blindsight, conscious vision, and the role of primary visual cortex. Prog. Brain Res. 155, 217-234.

(Van Gulick, 1989), the initiation of these responses cannot rely exclusively on the (unconscious, Velmans, 1991) neuronal processes that generate stimulus awareness. It is only because our blindsight-experienced patients unexpectedly responded much better than expected on the basis of random guessing to un-cued subjectively invisible stimuli that we can demonstrate that they required neither awareness of the stimuli nor perceptible cues to initiate their responses. In a variation on Paul Churchland's dictum (Churchland, 1984, p. 45-46) we conclude that not cued, but only cueless blindsight knocks 'the stuffing out of the "obvious" assumption that awareness of a signal is necessary for an intentional response to that signal'.

\section{ACKNOWLEDGMENT}

It is pleasure to thank the participants for their cooperation and both Eike A.Schmidt and Jürgen Seidel for valuable assistance.

Stoerig, P. (2008). Functional rehabilitation of partial cortical blindness? Restor. Neurol. Neurosci. 26 291-303.

Stoerig, P., and Barth, E. (2001) Phenomenal vision in a hemianopic field. Conscious. Cogn. 10, 574-587.

Stoerig, P., and Cowey, A. (1989). Wavelength sensitivity in blindsight. Nature 342, 916-918.

Stoerig, P., and Cowey, A. (1997) Blindsight in man and monkey. Brain 120, 535-559.

Stoerig, P., Zontanou, A., and Cowey, A. (2002). Aware or unaware? Assessment of cortical blindness in four men and monkey. Cereb. Cortex $12,565-574$.

Teuber,H.-L., Battersby, W.S., and Bender, M. B. (1960). Visual Field Defects after Penetrating Missile Wounds. Cambridge, MA, Harvard University Press.

Tong, F. (2003). Primary visual cortex and visual awareness. Nat. Rev. Neurosci. 4, 219-229.

Van Gulick, R. (1989). What difference does consciousness make? Philos. Top. 17, 211-230.

Velmans, M. (1991). Is human information processing conscious? Behav. Brain Sci. 14, 651-701.

Weiskrantz, L. (1986). Blindsight: A Case Study and its Implications. Oxford, Oxford University Press.

Weiskrantz, L., Barbur, J. L., and Sahraie, A. (1995). Parameters affecting conscious versus unconscious visual discrimination with damage to the visual cortex (V1). Proc. Natl. Acad. Sci. U.S.A. 92, 6122-6126.

Weiskrantz, L., Cowey, A., and Barbur J. L. (1999). Differential pupillary constriction and awareness in the absence of striate cortex. Brain 122, 1533-1538.
Weiskrantz, L., Warrington, E. K., Sanders, M. D., and Marshall, J. (1974). Visual capacity in the hemianopic field following a restricted occipital ablation. Brain 97, 709-728.

Wilcoxon, F. (1945). Individual comparisons by ranking methods. Biometrics $1,80-83$.

Zeki, S. (2001). Localization and globalization in conscious vision. Annu. Rev. Neurosci. 24, 57-86.

Zeki, S., and Ffytche, D. H. (1998). The Riddoch syndrome: insights into the neurobiology of conscious vision. Brain 121, 125-145.

Zihl, J. (1980). “Blindsight”: improvement of visually guided eye movements by systematic practice in patients with cerebral blindness. Neuropsychologia 18, 71-77.

Zihl, J., Tretter, F., and Singer, W. (1980). Phasic electrodermal responses after visual stimulation in the cortically blind hemifield. Behav. Brain Res. 1, 197-203.

Conflict of Interest Statement: The authors declare that the research was conducted in the absence of any commercial or financial relationships that could be construed as a potential conflict of interest.

Received: 03 September 2009; paper pending published: 22 October 2009; accepted: 17 December 2009; published online: 04 January 2010.

Citation: Stoerig P (2010) Cueless blindsight. Front. Hum. Neurosci. 3:74. doi: 10.3389/neuro.09.074.2009

Copyright (c) 2010 Stoerig. This is an openaccess article subject to an exclusive license agreement between the authors and the Frontiers Research Foundation, which permits unrestricted use, distribution, and reproduction in any medium, provided the original authors and source are credited. 